\title{
Incidence des acides gras alimentaires à chaîne moyenne, incorporés dans le régime de transition en post-sevrage, sur les caractéristiques de croissance et de carcasse d'agneaux sevrés précocement et engraissés intensivement en bergerie en été
}

\author{
G Bozzolo 1, M Bouillier-Oudot 1, M Aoun 1 \\ avec la collaboration technique de D Grasset ${ }^{2}, \mathrm{H}_{\text {Manse }}{ }^{1}$ \\ 1 ENSAT (École nationale supérieure agronomique de Toulouse), laboratoire de zootechnie \\ 31076 Toulouse Cedex; \\ 2 GEBRO (Groupement des éleveurs de brebis du bassin de Roquefort) 12101 Millau cedex, France
}

(Reçu le 12 novembre 1990; accepté le 6 mars 1991)

\begin{abstract}
Résumé - L'incidence de l'incorporation de matières grasses riches en acides gras à chaîne moyenne (huile de coprah) dans le régime de transition de post-sevrage sur les paramètres de croissance et les caractéristiques de carcasses d'agneaux de boucherie a été examinée sur 428 agneaux mâles de race Lacaune, sevrés précocement et engraissés intensivement en bergerie, durant l'été. Deux échantillons normalisés d'agneaux ( $n=144$ et $n=112$ ), l'un composé d'individus pesant à l'entrée en engraissement entre 9,5 et $14 \mathrm{~kg}$, l'autre distinguant les agneaux légers $(9-11 \mathrm{~kg})$ et les agneaux lourds $(12-15 \mathrm{~kg})$ ont été nourris avec 4 formulations d'aliment de démarrage différentes, pendant 3 semaines. Deux régimes (ME) de concentration énergétique égale à $1 \mathrm{UFV} / \mathrm{kg}$ MS, 2 autres (HE) à plus forte concentration énergétique (1,05 UFV et 1,12 UFV/kg de MS) ont permis d'analyser l'influence du niveau énergétique. Pour chacune de ces composantes, un aliment était enrichi avec de l'huile de coprah à raison de $5 \%$ pour tester la qualité de l'énergie apportée en partie sous forme d'acides gras à chaîne moyenne, l'ensemble présentant des valeurs isoazotées (190 $\mathrm{g}$ de MAT). La poursuite de l'engraissement s'est réalisée avec un aliment commercial de finition (1 UFV/kg MS, $165 \mathrm{~g}$ de MAT/kg de MS) jusqu'à atteindre le poids d'abattage de $39 \mathrm{~kg}$. Par comparaison avec des agneaux engraissés en fin d'hiver, dans les mêmes conditions, l'effet saison lié à la chaleur est marqué. Il se traduit par une diminution des performances de croissance (GMQ : $280 \mathrm{~g} / \mathrm{j}$ contre $298 \mathrm{~g} / \mathrm{j}$ : durée d'engraissement : 102 j contre 91 j) et une amélioration de la tenue et couleur des carcasses. Sur l'ensemble de l'engraissement, l'apparente régularité dégagée par le GMQ global et la durée d'engraissement dans le premier échantillon, masque des disparités liées aux régimes dans les séquences de croissance. Le second échantillon met en évidence un effet du poids des animaux au sevrage qui interfère avec l'influence des régimes. L'aliment ME enrichi en huile de coprah permet une bonne croissance des agneaux légers pendant la phase de tran-sition (193 g/j contre $96 \mathrm{~g} / \mathrm{j})$. Ce même aliment au coprah assure une plus grande régularité du profil de croissance des agneaux. Les qualités de carcasse : tenue, couleur, sont sensibles à l'effet différé des régimes de démarrage. La fermeté des gras de carcasse est moins bonne avec l'aliment ME témoin (NTEN : 3,5) qu'avec les 3 autres (NTEN > 4). Les individus qui développent un GMQ important en phase terminale d'engraissement ( $60 \mathrm{j}$ - abattage), possèdent des dépôts gras de meilleure qualité, même lorsqu'il s'agit de l'accomplissement d'une croissance compensatrice. Les régimes à forle concentration énergétique ne permettent pas de réduire la souffrance énergétique des agneaux en début d'engraissement (manque d'appétence); par contre, ils induisent un plus fort engraissement interne chez les agneaux légers au départ.
\end{abstract}


Summary - Influence of the incorporation of medium-chain fatty acids in post-weaning diet upon growth scores and carcass characteristics of early-weaned lambs intensively fattened in the pen during summer. The influence of the incorporation of medium-chain fatty acids (coconut oil) in the transition diet upon growth scores and carcass characteristics of market lambs, when given after weaning, was examined in dairy Lacaune male lambs, which had been weaned early at 5 wk and intensively fattened in a pen during the summer. Two standardized samples of lambs $(\mathrm{n}=144$ and $\mathrm{n}$ $=122$ ) were used, taking into account the initial weight at the beginning of the fattening period. The first sample consisted of standardized lambs weighing $9.5-14 \mathrm{~kg}$, and the second light and heavy lambs (9-11 $\mathrm{kg}$ and $12-15 \mathrm{~kg}$, respectively). The animals were fed with 4 different diets over a 3-wk period. Two diets with an energy concentration of $1.0 \mathrm{UFV} / \mathrm{kg} D M(M E)$ and 2 with a higher energy concentration (HE: $1.05 \mathrm{UFV}$ and $1.12 \mathrm{UFV} / \mathrm{kg}$ DM) were used to test the influence of energetic status. The influence of medium-chain fatty acids was analyzed with the introduction of $5 \%$ coconut oil in one diet of every level of energy. All the diets had the same level of crude protein (190 g/kg DM). The late fattening period included the same commercial diet (NE =1 UFV/kg DM and CP = $165 \mathrm{~g} / \mathrm{kg} \mathrm{DM}$ ) to a slaughtering weight of $39 \mathrm{~kg}$. Comparison with a sample studied at the end of the previous winter showed a reduction in growth scores (DGR: 280 vs $298 \mathrm{~g}$; fattening duration: 102 vs $91 \mathrm{~d}$ ) and improved firmness and color of the carcasses. Regarding overall fattening, the apparent similarity between growth rate and fattening duration hides differences in the growth sequences. The second sample shows the influence of diets and weaning weight upon growth curves. The daily growth rate of the light lambs fed with diet ME supplemented with coconut oil is higher than the 3 others during the transition period (193 g/d vs $96 \mathrm{~g} / d$ ), and the growth curve is significantly smoother. The chilled carcasses obtained with the HE diets and the ME + coconut oil diet are firmer than those obtained with the ME reference diet (NTEN > 4 vS NTEN = 3.5). It is clear that the higher the growth rate of the lambs through the late fattening period ( $60 \mathrm{~d}$ to slaughtering) the better the fat qualities are, even if they exhibit a compensatory growth. The highest energetic concentration diets do not reduce the energetic deficiency at the beginning of the fattening period (post-weaning period) but bring about a higher internal fat increase in the lightest lambs at the onset of the post-weaning period.

market lamb / coconut oil / growth / carcasses

\section{INTRODUCTION}

La mise en évidence d'un effet négatif exercé par une forte vitesse de croissance sur la fermeté des gras de carcasse d'agneaux (Aurousseau et al, 1973), notamment chez les mâles lorsque ceux-ci sont engraissés avec un aliment à forte concentration énergétique après avoir été sevrés précocement (4-5 semaines) (Bozzolo et al, 1990), pose le problème de l'incidence d'une manipulation alimentaire sur la quantité et la qualité du tissu adipeux des carcasses (couleur et consistance). Cette action pourrait prendre effet dès le début de la phase d'engraissement, au sortir de l'allaitement maternel, avec la distribution de l'aliment de démarrage. En effet, ces jeunes néoruminants encore proches d'une physiologie digestive de type monogastrique subissent, lorsqu'ils sont sevrés tôt, une forte crise de déficit énergétique, le temps de leur adaptation à un régime solide. Celle-ci se cumule d'ailleurs avec les effets de stress occasionnés par la séparation, le transport et les allotements réalisés dans les centres d'engraissement.

La conséquence immédiate est d'induire l'utilisation d'acides aminés à des fins glucogéniques, et de provoquer une lipolyse accentuée pour pallier une ingestion d'aliments solides déficiente (Dantzer et Mormède, 1983; Annison, 1984; Shetty, 1990). Cette souffrance de post-sevrage, comparable dans ses conséquences aux réac- 
tions néonatales, pourrait conduire ultérieurement à une croissance compensatrice. Or, les effets de celle-ci se traduisent par un anabolisme protidique élevé, accompagnant des teneurs en eau accrues dans la carcasse au détriment des lipides (Ferrel et al, 1986; Turgeon et al, 1986).

Par ailleurs, la concentration énergétique et la nature de l'énergie proposée dans la ration peuvent influencer la régularité de la courbe de croissance ainsi que la qualité du tissu gras (Asplund et al, 1985; Waghorn et al, 1987; Abdul Razzaq et Bickerstaffe, 1989). Aurousseau et al (1973), montrent une incidence différée des acides gras à chaîne moyenne lorsqu'ils sont introduits dans un aliment d'allaitement. Par ailleurs, chez le porcelet, Benevenga et al (1989), Odle et al (1989) constatent, quand les animaux sont supplémentés avec des acides gras courts à nombre pair d'atomes de carbone, un effet d'épargne des acides aminés, rejoignant les conclusions de Sutton et al (1983), Aurousseau et al (1989b), à propos de l'agneau en croissance.

En revanche, chez l'agneau ruminant, le rôle des acides gras mono-insaturés, ramifiés et impairs, issus du métabolisme de l'acide propionique souvent produit en excès avec des rations fortement énergétiques et amylacées, est néfaste à la tenue des carcasses (Garton et al, 1972; Aurousseau, 1981; Busboom et al, 1981). Limiter la surcharge propionique tout en épargnant les acides aminés engagés dans la voie glucogénique permettrait-il de réduire le risque de générer les acides gras incriminés dans ce défaut de carcasse ?

Les acides gras peuvent, en ce sens, constituer un substitut énergétique efficace à condition qu'ils soient très digestibles et à faible nombre d'atomes de carbone afin d'être préférentiellement catabolisés dans le foie et les muscles plutôt que d'être orientés vers la lipogenèse (Frost et Wells,
1981). Le choix d'une telle supplémentation énergétique paraît justifié en raison de la densité calorique élevée des lipides. De plus, ceux-ci sont notablement représentés dans l'alimentation maternelle antérieure environ $55 \%$ de la valeur énergétique du lait de brebis Lacaune d'après les données de Van Quackebeke et al (1981) et Cottier et Liquière (1986) - impliquant un équipement enzymatique digestif déjà adapté à ce type de constituant alimentaire alors que celui-ci est encore peu fonctionnel concernant l'utilisation des amidons (Leat et Harrison, 1975).

En contrepartie, la forte valeur énergétique des lipides permet d'accroitre la proportion de cellulose dans la formulation des aliments. Cette possibilité offre une garantie supplémentaire de meilleur fonctionnement du rumen en phase d'initiation de l'activité fermentaire $(\mathrm{pH}$, vitesse de transit) et permet sans doute de réduire la part des acides gras volatils précurseurs des acides gras responsables du manque de fermeté des carcasses.

Ce travail rapporte les résultats concernant la mise en application de ces hypothèses, testées sur des agneaux mâles Lacaune, sevrés précocement et engraissés intensivement en bergerie. Les effets sont analysés en rapport avec l'évolution des courbes de croissance et avec les caractéristiques des carcasses obtenues.

\section{MATÉRIEL ET MÉTHODES}

\section{Animaux et conduite de l'élevage}

Au total, 428 agneaux mâles de race Lacaune de souche laitière ont servi à la réalisation de cet essai. Ces animaux, sevrés précocement (moins de 35 j) ont été engraissés intensivement en bergerie (centre d'engraissement du GEBRO) à l'aide d'aliments granulés distribués à volonté avec un complément de paille mis à 
libre disposition. Deux types d'aliments ont été utilisés. L'un de préengraissement, adapté à la transition de post-sevrage, comporte un prémix antibio-supplémenté permettant de réduire les effets de stress et d'assurer une meilleure maîtrise sanitaire pour des sujets fragilisés; il a été distribué durant une période de plus de $21 \mathrm{j}$ jusqu'à concurrence de $17 \mathrm{~kg}$ en moyenne par agneau. L'autre, après une brève transition, a été fourni à volonté jusqu'au sacrifice et correspondait à un aliment d'engraissement commercial.

La stratégie d'abattage a été réalisée sur le principe de l'obtention d'un poids constant, dès lors que les agneaux dépassaient $39 \mathrm{~kg}$, à partir d'un tri hebdomadaire.

Ceux-vi ont été engraissés en été (mai à début septembre). Or, dans le bassin laitier de Roquefort, plus de $80 \%$ des naissances sont regroupées entre novembre et février, ce qui correspond à une production d'agneaux de fin d'hiver. Pour situer cet essai par rapport à une référence, nous avons donc comparé les résultats principaux à ceux obtenus en début de saison (janvier à mi-mai) avec des agneaux of ( $n$ $=487$ ) issus du schéma de sélection Lacauneviande poursuivi par le GEBRO. Ces derniers provenaient du croisement $\sigma^{*}$ souche Lacauneviande $x \&$ primipares souche Lacaune-lait et furent conduits de la même façon, sur le même lieu. L'ensemble des relevés, notamment l'appréciation des caractéristiques de carcasse, a été mené par le même opérateur.

\section{Aliments}

C'est sur l'influence de l'aliment de démarrage que portent les présents résultats. Quatre formulations ont été comparées pour tenter de cerner l'incidence du niveau énergétique de la ration d'une part, et la qualité de l'énergie ingérée d'autre part, sur la croissance et les caractéristiques de carcasse.

Deux régimes (Alim 1 et Alim $_{2}$ ) possèdent une concentration énergétique de : $1 \mathrm{UFV} / \mathrm{kg}$ MS (ME). Deux autres (Alim 3 et Alim 4 ) ont une concentration plus élevée (HE : 1,05 UFV et $1,12 \mathrm{UFV} / \mathrm{kg} \mathrm{MS}$ ), l'ensemble étant ajusté sur une base iso-azotée. Pour chacune de ces variantes de niveau d'apport énergétique, l'introduction d'acides gras à chaîne moyenne a été réalisée en incorporant $5 \%$ d'huile de coprah dans l'un des aliments (aliments 2 et 4 respectivement). Ce taux a été choisi pour éviter de dépasser $7 \%$ de lipides dans la ration et risquer une diminution éventuelle de l'appétit des animaux (Sutton et al, 1983). La composition chimique et la valeur nutritive sont présentées dans le tableau la et leur composition en acides gras dans le tableau lb. Leur valeur énergétique exprimée en UFV a été calculée à partir de l'équation de prédiction proposée par Sauvant (1981) et la composition en acides gras a été déterminée par chromatographie en phase gazeuse, après préparation de leurs esters méthyliques selon la méthode AFNOR (NFT 60-233).

Conformément à l'objectif de fabrication, les régimes 2 et 4 contiennent un taux global de lipides environ 3 fois plus élevé que ceux des autres aliments comparés. Par ailleurs, la composition de la matière grasse pour ces 2 régimes fait ressortir la forte participation des $A G$ saturés à faible nombre de carbones, issus de l'huile de coprah, par rapport aux témoins non enrichis ( $\mathrm{nb}$ de $\mathrm{C}<14$ : > 50\% vs $<2 \%$ ). Leur proportion en acide oléique est moitié moindre et celle d'acide linoléique réduite (18 et $22 \%$ contre $58 \%$ ). Ainsi l'énergie brute contenue dans les lipides représente $14 \%$ de l'énergie totale pour les régimes enrichis en huile de coprah contre $5 \%$ pour les régimes témoins (1 et 3). La composition en AG des lipides de ces 2 derniers reflète leur origine céréalière.

Concernant la proportion en cellulose brute des régimes, celle-ci est nettement supérieure dans l'aliment $2(12 \%$ contre $6 \%$ pour les aliments 3 et 4 , et $8 \%$ pour le régime témoin $M E$ ).

La teneur en MAT indique bien une concentration iso-azotée pour les divers aliments de démarrage testés.

\section{Paramètres zootechniques mesurés}

Deux groupes d'observations ont été relevés :

- des pesées régulièrement espacées tous les 20 j jusqu'à l'abattage ont été effectuées pour suivre la croissance des agneaux, soient 5 mesures : $P_{\mathrm{EN}}, P_{20}, P_{40}, P_{60}, \mathrm{P}_{\mathrm{ABA}}$, pour les poids à l'entrée en engraissement, après $20 \mathrm{j}$, $40 \mathrm{j}, 60 \mathrm{j}$ d'engraissement et le poids à l'abattage. Les GMQ (gain moyen quotidien) correspondants $\left(G M Q_{\mathrm{E}-20}, G M Q_{20-40}, G M Q_{40-60}\right.$, 
Tableau la. Composition chimique et valeur nutritive des différents régimes expérimentaux.

\begin{tabular}{|c|c|c|c|c|c|}
\hline Composition & $\begin{array}{c}\text { Aliment } 1 \\
\text { (témoin ME) }\end{array}$ & $\begin{array}{c}\text { Aliment } 2 \\
\text { (coprah ME) }\end{array}$ & $\begin{array}{r}\text { Aliment } 3 \\
\text { (témoin HE) }\end{array}$ & $\begin{array}{c}\text { Aliment } 4 \\
\text { (corpah HE) }\end{array}$ & $\begin{array}{l}\text { Aliment } \\
\text { finition }\end{array}$ \\
\hline Mat sèche \% & 91,3 & 92,1 & 91,1 & 92,1 & 90,9 \\
\hline Mat minerale (\%MS) & 9,2 & 8,9 & 7,1 & 6,4 & 8,5 \\
\hline Mat cellulosique (\%MS) & 8,0 & 11,8 & 6,0 & 6,5 & 8,0 \\
\hline Lignine (\%MS) & 2,4 & 3,8 & 2,2 & 2,0 & 2,4 \\
\hline Mat protéique brute (\%MS) & 18,9 & 18.9 & 19,2 & 18,6 & 16,4 \\
\hline Mat grasse (\%MS) & 2,2 & 6,8 & 2,1 & 6,4 & 3,0 \\
\hline UFV/kg Mat sèche & 1,00 & 1,00 & 1,05 & 1,12 & 1.02 \\
\hline UFV/kg Mat brute & 0,92 & 0,92 & 0,96 & 1,03 & 0,93 \\
\hline EM en Mcal $/ \mathrm{kg}$ Mat sèche & 2,69 & 2,69 & 2,82 & 3,01 & 2,74 \\
\hline
\end{tabular}

Tableau lb. Composition en acides gras de différents aliments (en \% du poids des esters méthyliques totaux).

\begin{tabular}{lccccc}
\hline $\begin{array}{c}\text { Acides gras } \\
\text { (\%) }\end{array}$ & $\begin{array}{c}\text { Alim 1 } \\
\text { (témoin ME) }\end{array}$ & $\begin{array}{c}\text { Alim 2 } \\
\text { (coprah.ME) }\end{array}$ & $\begin{array}{c}\text { Alim 3 } \\
\text { (témoin HE) }\end{array}$ & $\begin{array}{c}\text { Alim 4 } \\
\text { (coprah HE) }\end{array}$ & $\begin{array}{c}\text { Alim } \\
\text { finition }\end{array}$ \\
\hline C8 $=0$ & - & 3,12 & - & 3,73 & - \\
C10 $=0$ & - & 3,07 & - & 3,66 & - \\
C12 $=0$ & 1,21 & 36,35 & - & 31,85 & 1,39 \\
C14 $=0$ & 0,7 & 13,82 & 0,25 & 12,42 & 1,19 \\
C14 $=1$ & - & - & 0,14 & 0,14 & 0,77 \\
C16 $=0$ & 14,57 & 11,35 & 17,01 & 11,88 & 17,88 \\
C16 $=1$ & 2,15 & 0,17 & 0,37 & 0,17 & 1,33 \\
C17 $=0$ & 0,23 & 0,19 & - & - & 0,25 \\
C18 $=0$ & 1,83 & $2,3 \%$ & 2,36 & 2,13 & 5,17 \\
C18 $=1$ & 18,89 & 9,89 & 16,98 & 9,77 & 25,17 \\
C18 $=2$ & 57,78 & 18,00 & 57,65 & 22,21 & 42,95 \\
C18 $=3$ & 3,81 & 1,57 & 5,06 & 1,95 & 3,54 \\
C20 $=0$ & 0,15 & 0,04 & 0,10 & 0,07 & 0,13 \\
C20 $=1$ & 0,17 & 0,09 & 0,18 & 0,07 & 0,23 \\
\hline
\end{tabular}

$\left.G M Q_{60-A B A}\right)$ et la durée d'engraissement (DUEN) ont été calculés.

Les observations relatives aux carcasses ont été appréciées après $18 \mathrm{~h}$ de ressuage en chambre frigorifique à $4{ }^{\circ} \mathrm{C}$. Celles-ci ont porté sur les paramètres suivants : poids carcasse (PCAR), rendement commercial $(R D T=P C A R /$ (PABA-5\%)), notes de conformation et de couverture de gras (NCONF, NGEXT), appréciées selon les critères de classement EUROPA. Des mesures complémentaires du degré d'adiposité concernent l'épaisseur du gras dorsal (EPGDO) mesurée en $\mathrm{mm}$ à partir d'une incision pratiquée latéralement au niveau des $4^{\theta}$ et $5^{\theta}$ vertèbres lombaires, l'importance du gras interne estimée par le poids de gras périrénal (NGRO) et le gras intercostal (NGICO). Deux appréciations relatives à la qualité du gras de couverture ont été effectuées : l'une jugeant de la tenue (NTEN) selon 5 classes (de 1 : gras mou et huileux à 5 : 
gras ferme et "caillés"), l'autre jugeant de la couleur selon 7 niveaux de teintes de base (de 1 : blanc à 7 : jaune brunâtre avec reflets verdâtres) conformément à un code défini par Bozzolo et al (1990).

La teneur en eau (MH) du tissu gras externe a été déterminée par dessication en étuve à partir de prélèvements de gras péricaudal, préalablement broyés à l'aide d'un broyeur à billes en présence d'azote liquide.

\section{Traitement des résultats}

Une première confrontation a montré que l'influence du poids à l'entrée en engraissement (PEN) sur les autres paramètres était sensiblement différente selon la nature du régime alimentaire. En conséquence, pour réduire cette source de variabilité, nous avons procédé à 2 types d'échantillonnage plutôt que d'appliquer un traitement par covariance. Le premier (échantillon standardisé) a pour but de constituer des lots d'agneaux aussi homogènes que possible quant à leur poids à Yentrée en station d'engraissement, pour les différents régimes testés sur la base d'effectifs équilibrés. 36 animaux par régime expérimental ont été retenus (soit 144 agneaux au total), constituant 3 classes contiguës comprises entre 9,5 et $14 \mathrm{~kg}$ de poids vif $(9,5-11 \mathrm{~kg} ; 11-12,5 \mathrm{~kg} ; 12,5-$ $14 \mathrm{~kg}$ ), représentant respectivement $1 / 6,2 / 3,1 /$ 6 des effectifs et de sorte qu'un équilibre soit respecté par rapport à leur moyenne de classe. Le second (échantillon marginal) a pour objectif de mettre en évidence une éventuelle interaction entre les régimes alimentaires et le poids d'entrée, faible ou élevé, les agneaux légers étant souvent issus de naissances multiples ou de mères primipares. Ces derniers ont été choisis par tirage aléatoire dans la classe 9-11 kg; de même, les agneaux lourds ont été sélectionnés dans la classe de poids $12-15 \mathrm{~kg}$, soit 14 individus par modalité (112 agneaux au total).

Les différents paramètres observés ont été analysés indépendamment selon un plan factoriel à effets fixes (régimes; régime et type d'agneaux, avec interaction) et à $n$ répétitions aléatoires (respectivement $n=36$ et $n=14$ ).

Concernant la comparaison des profils de croissance en fonction du temps et compte tenu de l'autocorrélation des différents $G M Q$, après contrôle de la non sphéricité des matrices de variance covariance (test de Barlett généralisé, d'après Dagnélie, 1977), nous avons utilisé la procédure décrite par Allen et al (1983). Celle-ci procède par ajustement polynômial des profils individuels (GMQ en fonction du temps d'engraissement) et les coefficients de régression relatifs aux degrés significatifs du polynôme sont soumis à l'analyse de la variance. La comparaison des moyennes est effectuée à l'aide du test de Newman-Keuls à $P<0,05$; les tendances significatives sont signalées jusqu'à $P<0,10$.

Les variables de nature ordinale (NTEN, NCOU) sont abordées sous l'angle de leur distribution d'échantilonnage (test de $\chi^{2}$ ), dès lors que les effectifs sont suffisants.

\section{RÉSULTATS}

\section{Position de l'essai par rapport à la référence constituée par des agneaux élevés en début de saison}

Les résultats du tableau lla font apparaître un rythme de croissance supérieur pour les agneaux engraissés en début de saison, ce qui se traduit par une durée d'engraissement allongée de $10 \mathrm{j}$ en moyenne pour les agneaux de l'essai d'été, compte tenu de la stratégie d'abattage à poids constant. L'observation des paramètres de carcasses fait ressortir essentiellement une meilleure tenue et une moins bonne conformation des carcasses pour un poids et un recouvrement de gras externe inférieurs chez les agneaux d'été. Plus précisément, la répartition des notes de tenue (tableau Ilb) montre une dispersion plus réduite du classement. Plus de la moitié d'entre elles $(60 \%)$ peut être qualifiée de bonne (classes 4 et 5) pour les agneaux de l'essai d'été, alors que pour les agneaux de printemps la majorité (64\%) est mal classée (classes 1, 2, 3). Concernant les notes de coloration, les carcasses claires (1 et 2) sont mieux représentées dans l'échantillon d'été. 
Tableau lla. Comparaison des caractéristiques générales de l'essai d'été avec celles des agneaux engraissés en fin d'hiver.

\begin{tabular}{|c|c|c|c|c|c|c|}
\hline Variables & \multicolumn{2}{|c|}{$\begin{array}{c}\text { Échantillon } \\
\text { d'été } \\
\mathrm{n}=258 \\
\text { (sd) }\end{array}$} & \multicolumn{2}{|c|}{$\begin{array}{c}\text { Échantillon de } \\
\text { fin d'hiver } \\
n=487 \\
\text { (sd) }\end{array}$} & \multirow[t]{2}{*}{$t$} & $\mathrm{~s}$ \\
\hline \multicolumn{6}{|c|}{ Paramètres d'élevage } & \\
\hline PEN $\quad(\mathrm{kg})$ & 11,4 & $(1,1)$ & 12,0 & $(1,9)$ & $-4,67$ & $\star \star \star \star$ \\
\hline GMQ & 280,4 & $(42,9)$ & 298,2 & $(57,1)$ & $-4,39$ & $* * *$ \\
\hline DUEN & 102 & (15) & 91 & $(18)$ & 8,39 & $\star \star \star *$ \\
\hline $\mathrm{P} 60 \quad(\mathrm{~kg})$ & 27,3 & $(3,9)$ & 30,2 & $(4,9)$ & $-8,22$ & $* * *$ \\
\hline \multicolumn{7}{|c|}{ Paramètres de carcasse } \\
\hline PCAR (kg) & 19,3 & $(1,0)$ & 20,2 & $(0,9)$ & $-12,49$ & $* \star \star$ \\
\hline NTEN & 3,9 & $(1,0)$ & 3,3 & $(0,9)$ & 8,33 & *** \\
\hline EPGTO $(\mathrm{mm})$ & 3,0 & $(1,9)$ & 2,5 & $(1,8)$ & 3,54 & $* * *$ \\
\hline NGEXT & 2,6 & $(0,6)$ & 3,2 & $(0,5)$ & $-14,52$ & $* * *$ \\
\hline NGRO & 275 & (83) & 283 & $(85)$ & $-1,23$ & NS \\
\hline NCONF & 3,0 & $(0,8)$ & 4,1 & $(2,2)$ & $-7,76$ & $* * *$ \\
\hline
\end{tabular}

$t: t$ de Student; $S$ : seuil de signification; ${ }^{* * *}: P<0,001 ; N S$ : non significatif.

Tableau Ilb. Comparaison des caractéristiques de carcasse de l'essai d'été avec celles des agneaux engraissés en fin d'hiver. Répartition des effectifs (\%) par classe de tenue et de couleur.

\begin{tabular}{lrrr}
\hline & $\begin{array}{c}\text { Échantillon } \\
\text { d'été } \\
\mathrm{n}=258\end{array}$ & $\begin{array}{c}S \\
\text { de fin d'hiver } \\
\mathrm{n}=487\end{array}$ \\
\hline Notes de tenue $(* * *)^{c}$ & & & \\
1 & 0,4 & $*$ & 2,4 \\
2 & 8,6 & $* *$ & 16,0 \\
3 & 31,5 & $* * *$ & 45,8 \\
4 & 26,1 & $\mathrm{NS}$ & 25,5 \\
5 & 33,5 & $* * *$ & 10,4 \\
Note de couleur $\left(^{* * *}\right)$ & & & \\
$1-2$ & 60,7 & $* *$ & 46,2 \\
$3-4$ & 29,6 & $\mathrm{NS}$ & 35,2 \\
5 & 2,7 & $\mathrm{NS}$ & 4,1 \\
6 & 1,2 & $\mathrm{NS}$ & 1,5 \\
7 & 5,8 & $* *$ & 13,0 \\
\hline
\end{tabular}

$C\left(^{* * *}\right)$ signification à $P<0,001$ test de Kolmogorov Smirnov (comparaison des distributions de 2 echantillons (colonnes)). $S$ signification des différences entre pourcentages pour une même modalité (test $|\Sigma|):{ }^{*} P$ $<0,05 ; " * P<0,01$; *** $P<0,001$; NS non significatif.
L'observation de la mortalité au cours de la durée d'engraissement (tableau III) indique une perte générale importante. Celle-ci est particulièrement accentuée entre 20 et 40 j d'engraissement, excepté pour le régime 2 (ME), enrichi en huile de coprah et consommé pour l'essentiel pendant la période précédente (E-20 j) (4\% contre $20 \%$ en moyenne). Les 2 lots soumis aux régimes $\mathrm{HE}$ paraissent également plus touchés entre 0 et 20 j ( $10 \%$ contre $4 \%)$.

\section{Effets des régimes de démarrage sur les paramètres d'élevage}

\section{Échantillon d'agneaux standardisés (144 agneaux)}

Les principaux résultats confrontés en analyse de variance sont rapportés dans le tableau IV, chacune des variables étant traitée séparément. 
Tableau III. Mortalité observée (\%) en cours d'engraissement selon les régimes de démarrage $(n=428)$.

\begin{tabular}{|c|c|c|c|c|c|}
\hline \multirow[t]{2}{*}{ Régimes } & \multicolumn{5}{|c|}{ Périodes } \\
\hline & $\begin{array}{c}0 \\
20 j\end{array}$ & $\begin{array}{l}20- \\
40 j\end{array}$ & $\begin{array}{l}40- \\
60 j\end{array}$ & $\begin{array}{c}60 j- \\
A B A\end{array}$ & Total \\
\hline $\begin{array}{l}\text { Aliment } 1 \\
\text { (témoin } M E \text { ) }\end{array}$ & $3,4^{b}$ & $16,7^{a}$ & $5,3^{a b}$ & $5,6^{\mathrm{a}}$ & 28,0 \\
\hline $\begin{array}{l}\text { Aliment } 2 \\
\text { (coprah } M E \text { ) }\end{array}$ & $4,0^{b}$ & $4,4^{b}$ & $4,6^{b}$ & $2,9^{a}$ & 15,2 \\
\hline $\begin{array}{l}\text { Aliment } 3 \\
\text { (témoin } H E \text { ) }\end{array}$ & $10,0^{a}$ & $23,5^{a}$ & $11,3^{a}$ & $5,5^{a}$ & 42,2 \\
\hline $\begin{array}{l}\text { Aliment } 4 \\
\text { (coprah } H E \text { ) }\end{array}$ & $10,1^{a}$ & $23,6^{a}$ & $6,2^{a b}$ & $5,3^{a}$ & 39,0 \\
\hline Total & 6.7 & 16,4 & 6,4 & 4,6 & 30,4 \\
\hline
\end{tabular}

$\mathrm{a}, \mathrm{b}$ : différence intra périodes à $P<0,05$ (test $\left.\chi^{2}, \mid \mathrm{Z}\right)$

Pour les paramètres d'élevage, l'influence des régimes de transition se fait ressentir sur les vitesses de croissance jusqu'à 60 j d'engraissement. Pendant la période de consommation effective (0-20 j), nous observons une tendance des aliments ME ( 1 et 2 ) (contraste significatif à $P<0,07)$ à induire une meilleure croissance, en particulier pour le régime 2 , enrichi en coprah. L'effet significatif dégagé exclusivement par le régime témoin ME (1) avec respectivement un plus fort et un plus faible $G M Q$ entre $20-40$ j et entre 40 $60 \mathrm{j}$, est la traduction d'un artéfact expérimental. Ce lot a bénéficié d'un surcroît d'aliment de démarrage (20 kg contre 17 $\mathrm{kg}$ en moyenne par agneau pour les autres lots), ce qui a pu différer son adaptation à l'aliment de finition. Dans l'ensemble, les durées d'engraissement sont identiques (100 j). De même, la vitesse de croissance moyenne sur la totalité de l'en- graissement reste semblable pour les différents lots $(282 \mathrm{~g} / \mathrm{j})$.

La comparaison des profils de croissance (tableau V) montre une plus grande régularité du régime 2 . Ce lot se démarque des autres pour l'ensemble des coefficients de régression des divers degrés du polynôme d'ajustement. En particulier la valeur du GMQ extrapolée à 0 j, qui peut donner un aperçu du degré de souffrance des animaux, dénote un amaigrissement avec l'aliment 1, alors que l'aliment 2 induit une moyenne satisfaisante. En dépit d'une extériorisation plus tardive, le GMQ maximum moyen enregistré par le lot 2 ne diffère pas des autres.

\section{Échantillon marginal (agneaux lourds et légers) (112 agneaux)}

Certains effets principaux et d'interaction interviennent significativement (tableaux VI et VIla).

Nous retrouvons l'influence positive du régime 2 en période $0-20 \mathrm{j}$, cette fois plus nettement marquée en raison d'une interaction améliorant la croissance des agneaux légers (193 g/j contre $58 \mathrm{~g} / \mathrm{j}$ pour les régimes 2 et 1 respectivement et une moyenne de $114 \mathrm{~g} / \mathrm{\jmath}$ pour les régimes $H E$ ). Par ailleurs, le $G M Q$ réalisé par les agneaux lourds n'est pas différent pour cette composante alimentaire, alors que le différentiel entre agneaux lourds et légers au départ est de $+64 \%$ pour les régimes $\mathrm{HE}$ et dépasse $+300 \%$ pour le régime 1 (témoin ME).

Cet effet semble se poursuivre dans la période ultérieure entre $20-40 \mathrm{j}$. Une croissance compensatrice se dessine pour les régimes autres que l'aliment 2.

Entre 40 et $60 \mathrm{j}$, nous remarquons une supériorité différée des régimes $\mathrm{HE}$ initiaux sur les autres, toutefois assez faible 
Tableau IV. Influence du régime alimentaire de démarrage sur les paramètres zootechniques d'un échantillon d'agneaux, standardisé en fonction du poids d'entrée.

\begin{tabular}{|c|c|c|c|c|c|c|c|c|c|}
\hline \multirow{2}{*}{$\begin{array}{l}\text { Variables } \\
\text { Paramètres d'éleva }\end{array}$} & \multirow{2}{*}{ age } & \multicolumn{2}{|c|}{$\begin{array}{c}\text { Alim } 1 \text { (cv\%) } \\
\text { témoin } \mathrm{ME} \\
(n=36)\end{array}$} & \multicolumn{2}{|c|}{$\begin{array}{c}\text { Alim } 2 \text { (cv\%) } \\
\text { coprah } \mathrm{ME} \\
(n=36)\end{array}$} & \multicolumn{2}{|c|}{$\begin{array}{c}\text { Alim } 3 \text { (cv\%) } \\
\text { témoin } \mathrm{HE} \\
(n=36)\end{array}$} & \multicolumn{2}{|c|}{$\begin{array}{c}\text { Alim } 4 \text { (cv\%) } \\
\text { coprah } \mathrm{HE} \\
(n=36)\end{array}$} \\
\hline & & & & & & & & & \\
\hline$P E N(\mathrm{~kg})$ & NS & 11,7 & (7) & 11,7 & (7) & 11,8 & (7) & 11,7 & (8) \\
\hline$P 20(\mathrm{~kg})$ & NS & 15,0 & (15) & 15,2 & (15) & 14,5 & (15) & 14,7 & (14) \\
\hline$P 40(\mathrm{~kg})$ & NS $(5,4 \%)$ & 22,4 & (16) & 20,7 & (14) & 20,5 & (14) & 21,1 & (15) \\
\hline$P 60(\mathrm{~kg})$ & NS & 28,3 & (13) & 27,2 & (13) & 27,6 & (12) & 27,9 & (14) \\
\hline GMQE-20 (g) & NS & 162,1 & (45) & 176,5 & (46) & 138,7 & (67) & 149,8 & (57) \\
\hline GMQ20-40 (g) & $* * \star$ & $370,1^{a}$ & (25) & $274,6^{b}$ & (26) & $299,7^{b}$ & (23) & $317,4^{b}$ & (27) \\
\hline GMQ40-60 (g) & $* \star$ & $293,9^{a}$ & (21) & $325,1^{a b}$ & (26) & $356,2^{b}$ & (19) & $341,1^{b}$ & (29) \\
\hline GMQ60-ABA (g) & NS & 305,0 & (16) & 313,6 & (23) & 296,7 & (27) & 303,8 & (18) \\
\hline GMQE-ABA (g) & NS & 288,9 & (11) & 279,9 & (15) & 276,0 & (17) & 284,4 & (15) \\
\hline$D U E N(j)$ & NS & 98,7 & (13) & 101,6 & (15) & 103,1 & (14) & 99,7 & (15) \\
\hline
\end{tabular}

Paramètres de carcasse

\begin{tabular}{|c|c|c|c|c|c|c|c|c|c|}
\hline$P C A R C(\mathrm{~kg})$ & NS $(6 \%)$ & 19,5 & (3) & 19,3 & (5) & 19,4 & (4) & 19,1 & (3) \\
\hline$R D T(\%)$ & NS & 51,6 & (3) & 51,3 & (4) & 51,6 & (3) & 50,7 & (3) \\
\hline NCONF & NS & 3,1 & (31) & 3,1 & (27) & 2,9 & (25) & 3,0 & (25) \\
\hline NGEXT & NS & 2,6 & (22) & 2,7 & (25) & 2,4 & (23) & 2,6 & (22) \\
\hline NGICO & NS & 2,8 & (22) & 2,7 & (27) & 2,8 & (22) & 2,9 & (19) \\
\hline$E P G D O(\mathrm{~mm})$ & NS $(7 \%)$ & 3,3 & (63) & 2,8 & (67) & 2,2 & (52) & 2,9 & (60) \\
\hline$N G R O(g)$ & NS & 263,9 & (38) & 263,9 & (25) & 277,8 & (27) & 265,3 & (27) \\
\hline NTEN & * & $3,5^{\mathrm{a}}$ & (29) & $4,1^{b}$ & (24) & $4,1^{b}$ & (26) & $4,0^{a b}$ & (24) \\
\hline NCOUL & NS & 2,9 & (71) & 2,7 & (65) & 3,0 & (61) & 2,2 & (61) \\
\hline$M H(\%)$ & & $21,9^{a}$ & (22) & $21,3^{a b}$ & (22) & $19,5^{\mathrm{ab}}$ & (21) & $19,1^{b}$ & (23) \\
\hline
\end{tabular}

$S$ : seuil de signification *: $P<0,05 ;{ }^{* *}: P<0,01 ; * * *: P<0,001$; NS : non significatif; NS $(6 \%)$ : tendance significative à $P<0,06 . a, b$ : différence significative à $P<0,05$ (Newman-Keuls).

$(+17 \%)$, essentiellement due au rattrapage réalisé par les agneaux légers au départ (+ $27 \%)$. Nous notons la très forte croissance des agneaux légers, nourris au démarrage avec le régime témoin $\mathrm{HE}(3)$, qui dépasse celle des agneaux lourds (366 g/j contre $347 \mathrm{~g} / \mathrm{j}$ ).

Entre $60 \mathrm{j}$ et l'abattage, les GMQ se sont équilibrés et paraissent indifférents à l'influence des aliments de démarrage ainsi qu'aux catégories de poids au départ. Ceci se traduit en particulier par la pour- suite d'une croissance compensatrice des agneaux légers des régimes 1 et 3 non complémentés avec du coprah.

Sur l'ensemble de l'engraissement, les gains réalisés paraissent globalement identiques, quelle que soit la nature de l'aliment comparé; par contre, l'influence du poids à l'entrée est significative mais de faible importance (+ $9 \%$ ), en faveur des agneaux lourds. Cet effet est paradoxalement masqué par la plus forte croissance enregistrée par les individus légers du lot 3 
Tableau V. Comparaison des profils de croissance des agneaux au travers des coefficients de l'ajustement polynomial selon la nature du régime et du poids vif au sevrage.

\begin{tabular}{|c|c|c|c|c|c|c|c|c|c|}
\hline \multirow[t]{2}{*}{ Coefficients } & \multicolumn{5}{|c|}{ Régimes alimentaires } & \multicolumn{3}{|c|}{ Poids au sevrage } & \multirow{2}{*}{$\begin{array}{l}\text { Inter- } \\
\text { action } \\
\quad S\end{array}$} \\
\hline & $S$ & Alim 1 & Alim 2 & Alim 3 & Alim 4 & $S$ & Légers & Lourds & \\
\hline \multicolumn{10}{|c|}{ Echantillon standard } \\
\hline & & $(n=36)$ & $(n=36)$ & $(n=36)$ & $(n=36)$ & & & & \\
\hline GMQEO & $\star \star \star \star$ & $-149,1^{c}$ & $111,6^{a}$ & $9,8^{b}$ & $-29,8^{b}$ & & & & \\
\hline$x$ & $* * *$ & $40,0^{a}$ & $7,0^{c}$ & $14,7^{b c}$ & $21,9^{b}$ & & & & \\
\hline$x^{2}\left(\times 10^{-2}\right)$ & $* * \star$ & $-95,9 c$ & $-4,7^{a}$ & $-18,6^{a b}$ & $-40,6^{b}$ & & & & \\
\hline$x^{3}\left(\times 10^{-4}\right)$ & $\star \star \star \star$ & $67,1^{a}$ & $-1,3^{b}$ & $6,1^{\mathrm{b}}$ & $22,7^{b}$ & & & & \\
\hline$G M Q_{\max }(g)$ & NS & 406,1 & 384,2 & 389,3 & 383,0 & & & & \\
\hline$t_{\max }(j)$ & $* * *$ & $41,6^{c}$ & $60,6^{a}$ & $54,5^{\mathrm{ab}}$ & $50,4^{b}$ & & & & \\
\hline \multicolumn{10}{|c|}{ Echantillon marginal } \\
\hline & & $(n=28)$ & $(n=28)$ & $(n=28)$ & $(n=28)$ & & $(n=56)$ & $(n=56)$ & $(n=14)$ \\
\hline GMQEO & *** & $-143,1^{b}$ & $117,3^{a}$ & $-70,4^{b}$ & $-96,6^{b}$ & * & $-92,5$ & $-3,9$ & $*$ \\
\hline$x$ & *** & $5,2^{a}$ & $2,2^{b}$ & $4,4^{a}$ & $4,8^{a}$ & NS & 4,5 & 3,8 & * \\
\hline$x^{2}\left(\times 10^{-2}\right)$ & **** & $24,1^{a}$ & $2,6^{c}$ & $12,3^{b}$ & $15,9^{b}$ & NS & 13,2 & 14,2 & NS \\
\hline$X^{3}\left(\times 10^{-4}\right)$ & $\star \star \star *$ & $-30,2^{c}$ & $-2,9^{a}$ & $-13,8^{b}$ & $-19,2^{b}$ & NS & $-15,2$ & $-17,9$ & NS \\
\hline$G M Q_{\max }(\mathrm{g})$ & $*$ & $407,2^{a}$ & $352,9^{b}$ & $393,3^{\text {ab }}$ & $394,3^{a b}$ & NS & 377,2 & 396,7 & NS \\
\hline$t_{\max }(j)$ & NS & 68,4 & 66,6 & 74,2 & 69,5 & NS & 71,2 & 68,2 & NS \\
\hline
\end{tabular}

GMQEO : valeur à l'origine du gain journalier estimé par le modèle; $S:$ seuil de signification *:P<0,05; ${ }^{\star \star}: P<$ 0,$01 ; \cdots *: P<0,001 ;$ NS : non significatif. $a, b, c$ : difference significative à $P<0,05$ (Newman Keuls).

HE (280 g/ $\mathrm{j}$ contre $265 \mathrm{~g} / \mathrm{j}$ pour les agneaux lourds).

Nous retrouvons au travers de la durée d'engraissement les précédentes caractéristiques de croissance, avec un allongement des temps de finition supérieur à $20 \mathrm{j}$ pour les agneaux légers nourris avec les aliments 1,2 et 4 , alors que le régime 3 induit une durée identique pour les 2 catégories (105 j). En conséquence, pour le régime 3 , la durée d'engraissement des agneaux lourds au départ est prolongée (105 j contre $93 \mathrm{j}$ en moyenne pour les autres lots) et inversement pour les agneaux légers.
Les profils de croissance renforcent les observations dégagées dans le premier échantillon (tableau V, fig 1a). L'interaction fait ressortir une pénalisation supplémentaire pour les agneaux légers dans le début de la période d'adaptation, notamment pour ceux du lot 1 alors que ceux nourris à l'huile de coprah (2) ne diffèrent pas des plus lourds (fig 1b). Pour cet échantillon constitué de cas extrêmes, l'effet du poids des agneaux n'intervient pas sur le niveau du maximum de la croissance tandis que, pris dans son ensemble, le régime 2 reste en retrait. Les dates d'apparition de ces maxima ne sont pas différentes en fonction des diverses modalités. 


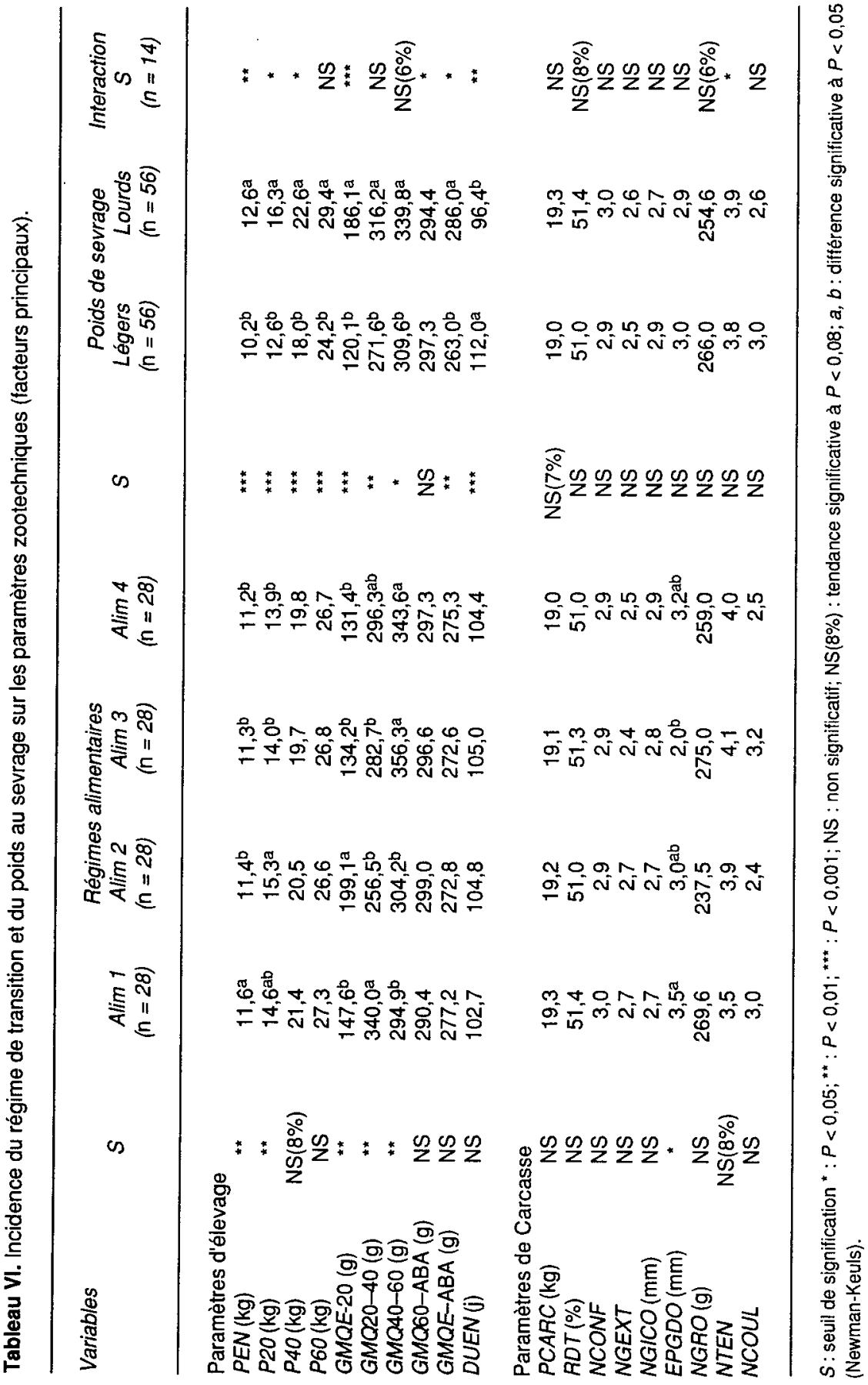


Tableau Vlla. Incidence du régime de transition et du poids au sevrage sur les paramètres d'élevage (interaction).

\begin{tabular}{|c|c|c|c|c|c|c|c|c|}
\hline \multirow{3}{*}{$\begin{array}{l}\text { Variables } \\
\text { (cv\%) }\end{array}$} & \multicolumn{8}{|c|}{ Régimes alimentaires / Classes de poids au sevrage } \\
\hline & \multicolumn{2}{|c|}{ Alim 1} & \multicolumn{2}{|c|}{ Alim 2} & \multicolumn{2}{|c|}{ Alim 3} & \multicolumn{2}{|c|}{ Alim 4} \\
\hline & $\begin{array}{l}\text { Légers } \\
(n=14)\end{array}$ & $\begin{array}{l}\text { Lourds } \\
(n=14)\end{array}$ & $\begin{array}{l}\text { Légers } \\
(\mathrm{n}=14)\end{array}$ & $\begin{array}{l}\text { Lourds } \\
(\mathrm{n}=14)\end{array}$ & $\begin{array}{l}\text { Légers } \\
(n=14)\end{array}$ & $\begin{array}{l}\text { Lourds } \\
(\mathrm{n}=14)\end{array}$ & $\begin{array}{l}\text { Légers } \\
(\mathrm{n}=14)\end{array}$ & $\begin{array}{l}\text { Lourds } \\
(n=14)\end{array}$ \\
\hline$P E N(\mathrm{~kg})$ & $\begin{array}{c}10,7^{\mathrm{b}} \\
\text { (4) }\end{array}$ & $\begin{array}{c}12,6^{a} \\
\text { (3) }\end{array}$ & $\begin{array}{c}10,0^{c} \\
(4)\end{array}$ & $\begin{array}{c}12,7^{\mathrm{a}} \\
(2)\end{array}$ & $\begin{array}{c}10,2^{\circ} \\
(7)\end{array}$ & $\begin{array}{c}12,5^{\mathrm{a}} \\
\text { (3) }\end{array}$ & $\begin{array}{r}9,9^{c} \\
(5)\end{array}$ & $\begin{array}{c}12,6^{\mathrm{a}} \\
(3)\end{array}$ \\
\hline$P 20(\mathrm{~kg})$ & $\begin{array}{c}11,9^{c} \\
(7)\end{array}$ & $\begin{array}{c}17,4^{\mathrm{a}} \\
(7)\end{array}$ & $\begin{array}{l}13,9^{d} \\
(15)\end{array}$ & $\begin{array}{l}16,8^{a b} \\
(6)\end{array}$ & $\begin{array}{l}12,5^{d} \\
(16)\end{array}$ & $\begin{array}{l}15,5^{\mathrm{b}} \\
(14)\end{array}$ & $\begin{array}{l}12,1^{d} \\
(18)\end{array}$ & $\begin{array}{c}15,6^{b} \\
(13)\end{array}$ \\
\hline$P 40(\mathrm{~kg})$ & $\begin{array}{c}17,8^{\mathrm{c}} \\
(13)\end{array}$ & $\begin{array}{c}25,0^{\mathrm{a}} \\
(10)\end{array}$ & $\begin{array}{r}18,9^{c} \\
(15)\end{array}$ & $\begin{array}{l}22,0^{b} \\
(10)\end{array}$ & $\begin{array}{l}18,0^{\circ} \\
(15)\end{array}$ & $\begin{array}{c}21,4^{b} \\
(16)\end{array}$ & $\begin{array}{c}17,4^{c} \\
(18)\end{array}$ & $\begin{array}{c}22,2^{b} \\
(14)\end{array}$ \\
\hline$P 60(\mathrm{~kg})$ & $\begin{array}{r}23,6 \\
(12)\end{array}$ & $\begin{array}{r}31,0 \\
(10)\end{array}$ & $\begin{array}{r}24,2 \\
(13)\end{array}$ & $\begin{array}{r}28,9 \\
(8)\end{array}$ & $\begin{array}{r}25,3 \\
(13)\end{array}$ & $\begin{array}{r}28,3 \\
(14)\end{array}$ & $\begin{array}{r}23,7 \\
(14)\end{array}$ & $\begin{array}{l}29,6 \\
(14)\end{array}$ \\
\hline GMQE-20 (g) & $\begin{array}{l}58,0^{d} \\
(52)\end{array}$ & $\begin{array}{l}237,2^{\mathrm{a}} \\
(17)\end{array}$ & $\begin{array}{l}193,5^{a b c} \\
(54)\end{array}$ & $\begin{array}{l}204,8^{a b} \\
(24)\end{array}$ & $\begin{array}{l}116,9^{\mathrm{cd}} \\
(68)\end{array}$ & $\begin{array}{l}151,5^{\mathrm{bc}} \\
(68)\end{array}$ & $\begin{array}{l}112,0^{\text {od }} \\
(94)\end{array}$ & $\begin{array}{l}150,7^{b c} \\
(64)\end{array}$ \\
\hline GMQ20-40 (g) & $\begin{array}{c}298,6 \\
(36)\end{array}$ & $\begin{array}{l}381,6 \\
(23)\end{array}$ & $\begin{array}{c}252,9 \\
(24)\end{array}$ & $\begin{array}{c}260,2 \\
(35)\end{array}$ & $\begin{array}{c}271,6 \\
(23)\end{array}$ & $\begin{array}{c}293,9 \\
(31)\end{array}$ & $\begin{array}{c}263,5 \\
(31)\end{array}$ & $\begin{array}{c}329,1 \\
(30)\end{array}$ \\
\hline GMQ40-60 (g) & $\begin{array}{c}290,7 \\
(30)\end{array}$ & $\begin{array}{c}299,2 \\
(22)\end{array}$ & $\begin{array}{c}264,6 \\
(29)\end{array}$ & $\begin{array}{c}343,8 \\
(25)\end{array}$ & $\begin{array}{c}365,8 \\
(16)\end{array}$ & $\begin{array}{c}346,8 \\
(18)\end{array}$ & $\begin{array}{c}317,4 \\
(22)\end{array}$ & $\begin{array}{l}369,7 \\
(19)\end{array}$ \\
\hline GMQ60-ABA (g) & $\begin{array}{c}301,9 \\
(20)\end{array}$ & $\begin{array}{c}278,8 \\
(15)\end{array}$ & $\begin{array}{c}286,6 \\
(19)\end{array}$ & $\begin{array}{c}311,3 \\
(13)\end{array}$ & $\begin{array}{c}318,8 \\
(18)\end{array}$ & $\begin{array}{c}274,4 \\
(33)\end{array}$ & $\begin{array}{l}281,6 \\
(16)\end{array}$ & $\begin{array}{l}313,0 \\
(21)\end{array}$ \\
\hline GMQE-ABA $(\mathrm{g})$ & $\begin{array}{c}255,7 \\
(11)\end{array}$ & $\begin{array}{c}298,8 \\
(13)\end{array}$ & $\begin{array}{l}260,7 \\
(17)\end{array}$ & $\begin{array}{c}285,0 \\
(8)\end{array}$ & $\begin{array}{l}280,0 \\
(16)\end{array}$ & $\begin{array}{c}265,2 \\
(21)\end{array}$ & $\begin{array}{l}255,6 \\
(15)\end{array}$ & $\begin{array}{c}295,1 \\
(17)\end{array}$ \\
\hline DUEN (j) & $\begin{array}{l}113,6^{a} \\
(10)\end{array}$ & $\begin{array}{l}91,8^{b} \\
(15)\end{array}$ & $\begin{array}{l}114,6^{\mathrm{a}} \\
(12)\end{array}$ & $\begin{array}{l}95,0^{\mathrm{b}} \\
(9)\end{array}$ & $\begin{array}{l}105,2^{a b} \\
(12)\end{array}$ & $\begin{array}{l}104,9^{a b} \\
(17)\end{array}$ & $\begin{array}{c}114,8^{a} \\
(9)\end{array}$ & $\begin{array}{c}93,9^{b} \\
(17)\end{array}$ \\
\hline
\end{tabular}

$a, b, c, d$ : difference significative à $P<0,05$ (Newman - Keuls).

\section{Effets des régimes de démarrage sur les paramètres de carcasse}

\section{Échantillon d'agneaux standardisés}

Les paramètres de carcasse (tableau IV) sont comparables, excepté la notation de la tenue qui est moins bonne pour le lot témoin ME (1), l'écart restant assez faible $(-0,5$ point $)$. L'analyse de la répartition des pourcentages d'effectifs selon les classes de tenue renforce cette observation (tableau VIlb).

L'examen des teneurs en eau $(M H) d u$ tissu gras péricaudal indique une valeur moyenne supérieure pour les agneaux soumis à l'aliment $1(22 \%)$ par rapport à l'aliment $4(19 \%)$, les autres régimes se situant en position intermédiaire. Le contraste réalisé entre niveaux énergétiques est aussi significatif. Toutefois les différences sont de faible ampleur ( 3 et $2 \%$ ).

\section{Échantillon marginal (agneaux lourds et légers)}

L'observation des résultats rapportés dans le tableau VIII indique que les influences sont modérées, voire absentes. Sur l'ensemble de l'essai, le poids au sevrage 

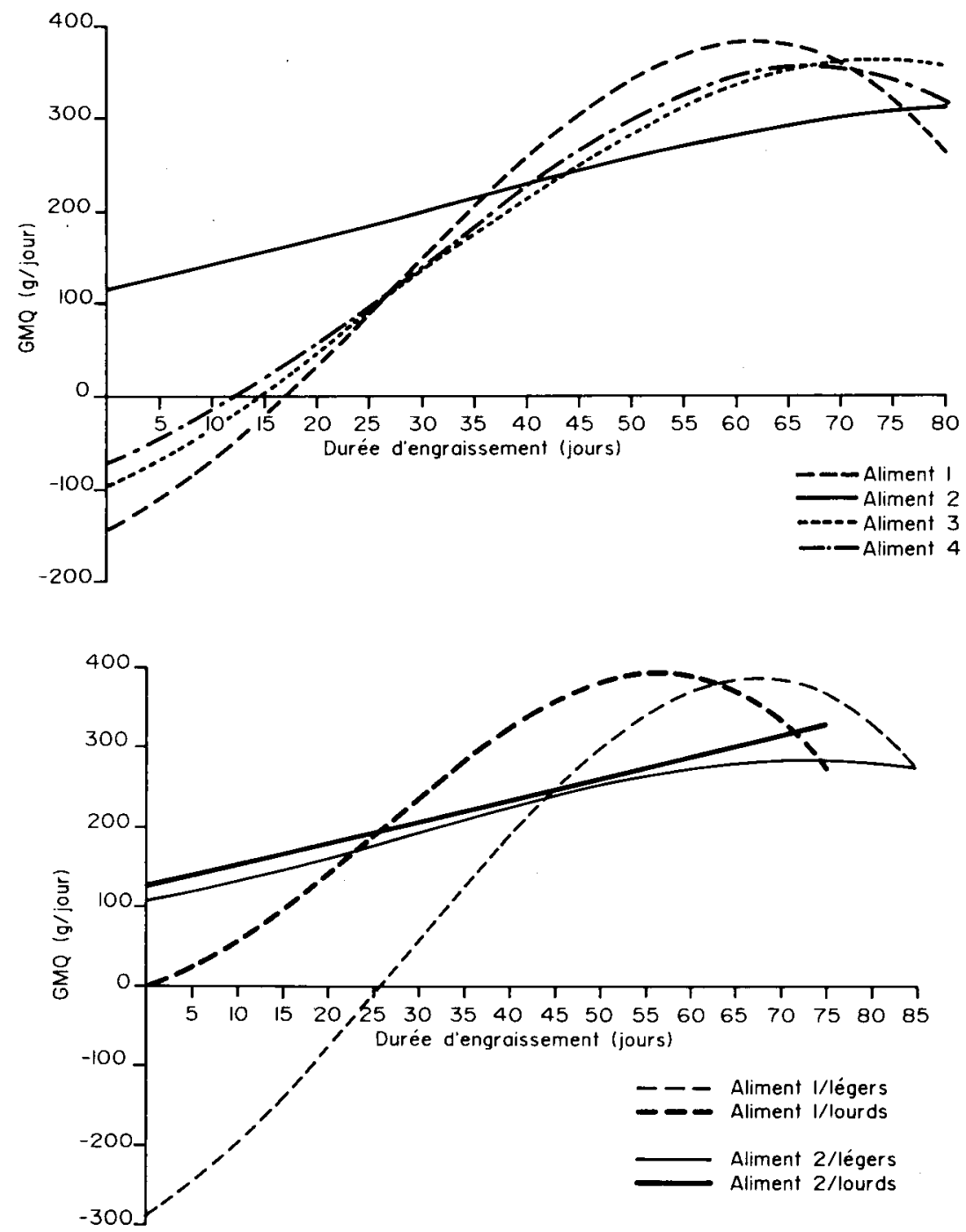

Fig 1. a. Profils de croissance selon les régimes de transition (ajustement polynômial - échantillon (standardisé). b. Profils de croissance en fonction du poids d'entrée en engraissement des régimes 1 (témoin ME) et 2 (coprah ME).

n'exerce pas d'influence sur les paramètres de carcasse en dehors d'une tendance $(P<0,07)$ sur le poids des carcasses. L'effet des régimes de démarrage intervient essentiellement sur l'épaisseur du gras dorsal, plus élevée chez les animaux nourris avec l'aliment 1 et plus faible pour ceux de l'aliment 3 . Les aliments enri- 
Tableau VIlb. Profils des effectifs (en \%) selon les classes de tenue de carcasse et le régime de démarrage (échantillon standardisé).

\begin{tabular}{llllll}
\hline Régime & & & & Tenue & \\
& 1 & 2 & 3 & 4 & 5
\end{tabular}

Aliment 1

(témoin ME) $\quad 2,8 \quad 11,1 \quad 36,1 \quad 33,3 \quad 16,7 \quad a$

Aliment 2

$\begin{array}{llllll}\text { (coprah ME) } & 0 & 5,6 & 27,8 & 19,4 & 47,2\end{array}$

Aliment 3

(témoin HE) $\quad 0 \quad 11,1 \quad 19,4 \quad 19,4 \quad 50,0 \quad b$

Aliment 4

$\begin{array}{llllll}\text { (coprah HE) } \quad 0 \quad 2,8 & 36,1 & 22,2 & 38,9 & \text { ab }\end{array}$

* $a, b$ différences significatives entre les profils lignes à $P<0,05$ (test de Kolmogorov - Smirnov).

chis en coprah sont situés en position intermédiaire.

Nous notons une tendance à la diminution de la note de tenue $(P<0,08)$ pour les agneaux nourris avec le régime 1 , d'ailleurs précisée par l'interaction significative $(P<0,05)$ avec les poids d'entrée. Les agneaux lourds ayant reçu de l'huile de coprah (lots 2 et 4 ) sont mieux notés que les légers, à l'inverse du lot 3 (HE). Le lot 1 (ME) ne traduit pas de différence entre catégories et reste le moins bien noté.

La faiblesse des effectifs à l'intérieur de chacune des modalités ( $n=14)$, ne permet pas d'envisager une comparaison sur les profils de répartition selon les classes de notation.

Une tendance à déposer plus de gras périrénal pour les agneaux légers démarrés avec les aliments HE apparaît $(287 \mathrm{~g}$ contre $246 \mathrm{~g}$ ), alors qu'avec le régime 2 les dépóts sont semblables. Pour le lot 1 , la tendance est inversée.

\section{DISCUSSION}

\section{Particularités des observations enregistrées dans l'essai}

Dans l'ensemble, les caractéristiques observées sur les agneaux engraissés dans cet essai au cours de l'été, traduisent des différences par rapport aux agneaux produits en début de saison. Deux manifestations principales semblent nettement marquées:

- les performances de croissance de ces agneaux sont plus faibles que celles réalisées par le groupe de fin d'hiver. À l'inverse, les qualités de carcasse, en particulier tenue et couleur, sont améliorées. Par ailleurs, la gamme des notations est plus restreinte par l'absence de carcasses présentant des dépôts adipeux très mous (TEN 1). La proportion élevée de carcasses bien classées ( $35 \%$ en TEN 5) est bien mise en évidence. Le même examinateur ayant opéré pour ces 2 sources de résultats, les différences observées pourraient être attribuées à des effets à la fois génétique et alimentaire. Concernant l'impact génétique, les agneaux de début de saison bénéficient des progrès apportés par le schéma de sélection Lacauneviande. Cependant, cet avantage ne peut être que modeste en raison du croisement réalisé sur primipares de la souche laitière. L'influence alimentaire n'est pas non plus suffisante pour expliquer ces écarts; la croissance réalisée avec le régime témoin 1 , commun aux 2 groupes, est moindre dans l'essai d'été. II s'agit là, vraisemblablement, d'une implication de la chaleur associée à la saison. Marchello et al (1967) ont déjà signalé l'influence positive des fortes températures sur la fermeté des gras externes des carcasses. De même, Busboom et al (1981) ont remarqué cet effet saison ainsi que Shelton et al (1972) 
Tableau VIII. Incidence du régime de transition et du poids au sevrage sur les paramètres de carcasse (interaction).

\begin{tabular}{|c|c|c|c|c|c|c|c|c|}
\hline \multirow{3}{*}{$\begin{array}{l}\text { Variables } \\
\text { (cv \%) }\end{array}$} & \multicolumn{8}{|c|}{ Régimes alimentaires / Classes de poids au sevrage } \\
\hline & \multicolumn{2}{|c|}{ Alim 1} & \multicolumn{2}{|c|}{ Alim 2} & \multicolumn{2}{|c|}{ Alim 3} & \multicolumn{2}{|c|}{ Alim 4} \\
\hline & $\begin{array}{l}\text { Légers } \\
\mathrm{n}=14\end{array}$ & $\begin{array}{l}\text { Lourds } \\
\mathrm{n}=14\end{array}$ & $\begin{array}{l}\text { Légers } \\
\mathrm{n}=14\end{array}$ & $\begin{array}{l}\text { Lourds } \\
n=14\end{array}$ & $\begin{array}{l}\text { Légers } \\
n=14\end{array}$ & $\begin{array}{l}\text { Lourds } \\
\mathrm{n}=14\end{array}$ & $\begin{array}{l}\text { Légers } \\
\mathrm{n}=14\end{array}$ & $\begin{array}{l}\text { Lourds } \\
\mathrm{n}=14\end{array}$ \\
\hline$P C A R(\mathrm{~kg})$ & $\begin{array}{c}19,1 \\
\text { (3) }\end{array}$ & $\begin{array}{l}19,4 \\
\text { (3) }\end{array}$ & $\begin{array}{c}19,0 \\
(5)\end{array}$ & $\begin{array}{c}19,4 \\
(4)\end{array}$ & $\begin{array}{l}18,8 \\
(5)\end{array}$ & $\begin{array}{c}19,4 \\
(4)\end{array}$ & $\begin{array}{c}19,0 \\
(5)\end{array}$ & $\begin{array}{r}18,9 \\
(4)\end{array}$ \\
\hline$R D T(\%)$ & $\begin{array}{l}51,1 \\
(3)\end{array}$ & $\begin{array}{l}51,8 \\
(3)\end{array}$ & $\begin{array}{l}50,9 \\
(4)\end{array}$ & $\begin{array}{l}51,6 \\
(4)\end{array}$ & $\begin{array}{l}50,8 \\
(4)\end{array}$ & $\begin{array}{l}51,9 \\
(4)\end{array}$ & $\begin{array}{l}51,6 \\
(3)\end{array}$ & $\begin{array}{l}50,3 \\
(4)\end{array}$ \\
\hline NCONF & $\begin{array}{c}2,9 \\
(35)\end{array}$ & $\begin{array}{c}3,1 \\
(24)\end{array}$ & $\begin{array}{c}2,8 \\
(30)\end{array}$ & $\begin{array}{c}3,1 \\
(22)\end{array}$ & $\begin{array}{c}3,1 \\
(20)\end{array}$ & $\begin{array}{l}2,8 \\
(28)\end{array}$ & $\begin{array}{c}3,0 \\
(21)\end{array}$ & $\begin{array}{c}2,9 \\
(31)\end{array}$ \\
\hline NGEXT & $\begin{array}{c}2,6 \\
(25)\end{array}$ & $\begin{array}{l}2,7 \\
(24)\end{array}$ & $\begin{array}{l}2,5 \\
(27)\end{array}$ & $\begin{array}{l}2,9 \\
(25)\end{array}$ & $\begin{array}{l}2,5 \\
(20)\end{array}$ & $\begin{array}{c}2,4 \\
(23)\end{array}$ & $\begin{array}{c}2,5 \\
(25)\end{array}$ & $\begin{array}{c}2,6 \\
(26)\end{array}$ \\
\hline NGICO & $\begin{array}{c}2,9 \\
(19)\end{array}$ & $\begin{array}{c}2,6 \\
(23)\end{array}$ & $\begin{array}{c}2,8 \\
(23)\end{array}$ & $\begin{array}{c}2,7 \\
(28)\end{array}$ & $\begin{array}{c}2,8 \\
(22)\end{array}$ & $\begin{array}{l}2,8 \\
(28)\end{array}$ & $\begin{array}{c}3,1 \\
(24)\end{array}$ & $\begin{array}{c}2,8 \\
(21)\end{array}$ \\
\hline$E P G D O(\mathrm{~mm})$ & $\begin{array}{c}3,8 \\
(66)\end{array}$ & $\begin{array}{c}3,3 \\
(54)\end{array}$ & $\begin{array}{c}2,6 \\
(64)\end{array}$ & $\begin{array}{c}3,5 \\
(67)\end{array}$ & $\begin{array}{c}2,1 \\
(40)\end{array}$ & $\begin{array}{c}1,9 \\
(52)\end{array}$ & $\begin{array}{c}3,5 \\
(83)\end{array}$ & $\begin{array}{c}2,9 \\
(60)\end{array}$ \\
\hline$N G R O(g)$ & $\begin{array}{l}257,1 \\
(27)\end{array}$ & $\begin{array}{c}282,1 \\
(25)\end{array}$ & $\begin{array}{c}232,1 \\
(32)\end{array}$ & $\begin{array}{l}242,9 \\
(20)\end{array}$ & $\begin{array}{l}285,7 \\
(20)\end{array}$ & $\begin{array}{l}264,3 \\
(16)\end{array}$ & $\begin{array}{l}289,3 \\
(25)\end{array}$ & $\begin{array}{l}228,6 \\
(27)\end{array}$ \\
\hline NTEN & $\begin{array}{l}3,4^{a} \\
(27)\end{array}$ & $\begin{array}{l}3,5^{a b} \\
(27)\end{array}$ & $\begin{array}{l}3,6^{a b} \\
(31)\end{array}$ & $\begin{array}{l}4, \gamma^{a b} \\
(21)\end{array}$ & $\begin{array}{l}4,5^{\mathrm{ab}} \\
(14)\end{array}$ & $\begin{array}{l}3,6^{b} \\
(37)\end{array}$ & $\begin{array}{l}3,8^{\mathrm{ab}} \\
(28)\end{array}$ & $\begin{array}{c}4,3^{\text {ab }} \\
(19)\end{array}$ \\
\hline NCOUL & $\begin{array}{c}2,7 \\
(62)\end{array}$ & $\begin{array}{c}3,1 \\
(74)\end{array}$ & $\begin{array}{c}3,0 \\
(55)\end{array}$ & $\begin{array}{c}1,8 \\
(70)\end{array}$ & $\begin{array}{c}3,3 \\
(63)\end{array}$ & $\begin{array}{c}3,1 \\
(66)\end{array}$ & $\begin{array}{c}2,6 \\
(48)\end{array}$ & $\begin{array}{c}2,3 \\
(74)\end{array}$ \\
\hline
\end{tabular}

a, b : différence significative à $P<0,05$ (Newman - Keuls).

traitant du rôle négatif de la tonte sur la fermeté des gras de carcasse. L'abaissement du métabolisme de base en réponse à la chaleur des bergeries, avec la diminution correspondante des taux plasmatiques de $\mathrm{GH}$, cortisol et surtout thyroxine (Beede et Collier, 1986) pourrait induire un ralentissement de l'activité métabolique du tissu adipeux (va-et-vient, lipogénèse-lipolyse) et favoriser ainsi l'accrétion des lipides dans les adipocytes à partir d'AG d'origine alimentaire. Ceci expliquerait aussi un rythme de croissance plus lent. La réduction de l'appétit et de l'efficacité alimentaire en réponse à la chaleur est bien connue chez les agneaux à l'engraissement (Ames et Brink, 1977; Schanbacher et al, 1982);
- la comptabilité des décès en cours d'engraissement fait ressortir une forte mortalité générale. L'accumulation des germes pathogènes en fin de saison dans les bergeries pourrait être une explication. En fait, la très forte mortalité enregistrée entre 20 et 40 j est due à une épidémie virale mal contrôlée d'echtyma. II est frappant d'observer l'absence de manifestation pour le lot alimenté avec le régime $2(\mathrm{ME})$, enrichi à l'huile de coprah. En raison du mode de transmission de cette maladie virale, il est difficile de conclure. Cependant, un meilleur approvisionnement énergétique pendant la période de fragilité (3 premières semaines) pourrait consolider l'immunité des agneaux. L'action antiseptique des 
acides laurique et myristique pourrait-elle réduire la contagion par voie digestive ? Cette observation mérite d'être contrôlée dans des essais futurs.

La mortalité supérieure enregistrée dans les lots 3 et 4 , nourris avec les régimes $\mathrm{HE}(10 \%$ contre $4 \%)$ peut traduire le résultat d'une mauvaise acceptation des aliments et d'une ingestion insuffisante chez les agneaux légers, catégorie la plus sensible au cours de cette période. L'ensemble des agneaux disparus correspondait en effet à des animaux qui pesaient moins de $10 \mathrm{~kg}$ à l'entrée.

\section{Incidence de l'incorporation d'huile de coprah dans l'aliment de démarrage sur les paramètres zootechniques - rôle de la concentration énergétique}

\section{Paramètres de croissance}

Durant la période soumise aux effets directs des régimes alimentaires $(0-20 \mathrm{j})$, la meilleure croissance enregistrée avec le régime 2 est très nettement marquée pour les agneaux légers. Cette action semble liée à l'efficacité de l'utilisation énergétique des acides gras à chaîne moyenne apportés par l'huile de coprah, lorsque les agneaux sont en situation de déficit comme l'ont montré d'autres auteurs, notamment chez le porcelet (Benevenga et al, 1989; Odle et al, 1989). Cette fraction lipidique représente plus de $6 \%$ de l'énergie brute dans les aliments enrichis en huile de coprah, soit environ $8 \%$ de l'énergie digestible mise à disposition en se rapportant aux coefficients de l'équation de prédiction citée par Giger-Reverdin et al (1990) et en considérant l'excellente digestibilité des acides gras à chaîne moyenne (Cera et al, 1989). Cette action spécifique sur les individus légers au sevrage peut s'expliquer par un besoin relatif en énergie supérieur pour les agneaux de faible poids. Leur métabolisme de base, étant proportionnel au poids élevé à une puissance inférieure à $1(0,73$ selon Taylor, (1980), Thompson et Parks (1985); 0,69+ $0,238 \times$ x $M Q$ selon Ferrel et al (1986)) reste, relativement à leur poids, plus élevé, alors que leurs réserves corporelles sont plus faibles que celles des agneaux lourds. Ces animaux réagiraient mieux à une élévation de la concentration énergétique de la ration sous réserve que ses composantes soient facilement assimilées et que l'appétence de l'aliment reste bonne. En outre, l'épargne azotée réalisée à ce stade et sa meilleure fixation (Sutton et al, 1983; Aurousseau et al, 1989a, 1989b) peuvent induire un anabolisme protidique important.

À l'inverse, l'influence exercée par le régime de préengraissement sur les agneaux lourds est inopérante. II pourrait s'agir aussi d'une meilleure préparation des agneaux lourds par complémentation avant le sevrage, ou d'animaux un peu plus âgés à l'entrée en bergerie, ou encore plus lourds à la naissance, autant de facteurs de variabilité non maîtrisés dans cet essai.

Dans l'ensemble, l'allure de la courbe de croissance est plus régulière et les phénomènes de croissance compensatrice sont estompés avec le régime ME coprah (2). C'est l'inverse avec les autres aliments, notamment exprimé par le lot 3 . Cependant, les effectifs d'agneaux nous semblent insuffisants pour compenser la forte variabilité intramodalité ( $c v=68 \%$ ), ce qui nous empêche de statuer pour ce régime. Ferrel et al (1986), examinant l'effet de divers niveaux de croissance durant la période de préengraissement (40 j), signalent une croissance compensatrice en rapport inverse avec la prise de poids initiale des agneaux et une activité métabolique intense. S'agissant d'une période 
de transition plus courte dans notre essai, avec des agneaux sevrés précocement, les effets que nous observons ne peuvent être aussi tranchés et avoir la même signification. Cependant, la tendance est semblable. En fait, l'effet majeur nous semble plutôt à rattacher à une meilleure capacité de réponse au stress par le biais d'un approvisionnement énergétique immédiatement disponible.

Il apparaît en conséquence, qu'en dépit d'une apparente neutralité de l'incidence des régimes alimentaires sur le GMQ couvrant toute la période d'engraissement, avec simplement une légère différence entre agneaux lourds et légers, une analyse plus fine fait ressortir de nombreuses différences et des effets compensateurs.

\section{Paramètres de carcasse}

Les régimes à forte concentration énergétique ou dotés en coprah, distribués en postsevrage, fournissent globalement de meilleures carcasses sur le plan de la tenue. Une orientation persistante vers la formation de lipides de réserve de type saturé pourrait s'exercer, comme l'ont suggéré Aurousseau et al (1973), à partir d'essais réalisés sur des agneaux nourris avec un aliment d'allaitement formulé avec de l'huile de coprah. II pourrait s'agir également d'un effet indirect d'épargne des acides gras saturés, antérieurement déposés dans les lipides corporels, moins sollicités en raison d'une lipolyse atténuée durant l'adaptation de post-sevrage, les agneaux recevant un aliment supplémenté en huile de coprah ne maigrissant pas dans cet essai. L'action de l'huile de coprah sur l'orientation des fermentations du rumen, diminuant les proportions des $A G V$ isobutyrique, butyrique, isovalérique (Sutton et al, 1983), initiateurs de chaînes d'acides gras ramifiés (Church, 1979), pourrait également induire un rôle favo- rable sur la tenue des gras de carcasse. Cependant, la moindre qualité des individus légers pour ce lot 2 laisse supposer, dans le cas des régimes plus énergétiques, une liaison positive entre les fortes vitesses de croissance dans la dernière période d'engraissement $(60 \mathrm{j}-A B A)$ et la fermeté du tissu gras, même lorsqu'il s'agit d'une croissance compensatrice comme semble le montrer le groupe des agneaux légers au départ, alimentés avec le régime 3. Turgeon et al (1986) suggèrent que les gains journaliers enregistrés au-delà de la deuxième moitié de l'engraissement ont une orientation nettement lipogénique, ce qui pourrait en partie expliquer cette interaction. D'ailleurs les agneaux qui réalisent à ce moment un fort croît déposent plus de gras interne (NGRO).

Enfin, il est probable que l'extériorisation de l'effet des régimes de démarrage sur la qualité du tissu adipeux peut rester très discrète en raison de l'implication précitée de la saison qui tend à réduire l'apparition des gras de très mauvaise qualité (TEN 1), mais aussi de l'imprécision qui demeure sur l'appréciation des classes intermédiaires de tenue. Cependant la teneur en eau mesurée sur les prélèvements paraît confirmer cette tendance.

\section{CONCLUSION}

Sous l'apparente uniformité des résultats d'engraissement globaux (GMQE-ABA, $D U E N)$, en plus de l'effet du poids des agneaux au sevrage, se cachent de nombreuses variations du rythme de croissance. Celles-ci font alterner ralentissements et croissances compensatrices, modulées par les incidences propres et différées des aliments de démarrage. Plusieurs arguments se conjuguent pour attirer l'attention sur l'intérêt d'incorporer de l'huile de coprah dans ces aliments de 
transition en post-sevrage. En dépit de nombreux facteurs non maîtrisés dans cet essai, nous mettons en évidence un rôle bénéfique des acides gras à chaîne moyenne pour les agneaux les plus légers, facilitant leur adaptation à l'alimentation solide. Seul l'aliment ME (1 UFV/kg de MS), formulé avec 5\% d'huile de coprah, a permis de réduire le déficit énergétique de post-sevrage de ces derniers. Cet effet se prolonge par l'induction d'un profil de croissance régulier, limitant les conséquences d'une forte croissance compensatrice.

Ce point mérite d'être approfondi, en particulier concernant son action sur les carcasses, car l'amélioration constatée est partagée avec les régimes plus concentrés. Le rôle réducteur de la saison sur l'apparition des carcasses flasques et huileuses, l'incertitude de la notation des classes intermédiaires de tenue peuvent contribuer à atténuer l'effet propre des facteurs analysés. Quoiqu'il en soit, l'incidence du préconditionnement alimentaire sur les effets de croissance compensatrice et leurs répercussions sur le tissu adipeux, bien que complexes, est très nette. Ceci ajoute à la conviction qu'une meilleure maîtrise du rationnement permettrait de progresser en proposant des régimes adaptés au potentiel génétique des agneaux. L'incidence sanitaire est également à considérer; notre observation en faveur de l'aliment ME enrichi au coprah demande à être confirmée car elle conditionne pour beaucoup la rentabilité de cette spéculation.

Enfin, dès lors que les agneaux sont bien démarrés, l'aliment de finition qui recouvre les $3 / 4$ de la durée d'engraissement est certainement plus influent sur la qualité des carcasses, la lipogénèse étant accélérée dans la phase terminale; il conviendrait donc de bien identifier les effets de ce dernier sur la qualité de l'accrétion adipeuse qu'il génère.

\section{RÉFÉRENCES}

Abdul-Razzaq HA, Bickerstaffe R (1969) The influence of rumen volatile fatty acids on protein metabolism in growing lambs. Br $J$ Nutr 62, 297-310

Allen OB, Burton JH, Holt JD (1983) Analysis of repeated measurements from animal experiments using polynomial regression. J Anim Sci $57,765-770$

Ames DR, Brink DR (1977) Effect of temperature on lamb performance and protein efficiency ratio. J Anim Sci 44, 136-140

Annison EF (1984) The metabolism of neutral and acidic lipids by tissues of the ruminant. In: Herbivore Nutrition in Subtropics and Tropics (Gilchrist FM, Mackie RI, eds) The Science Press, Publ, Craighall, South Africa 549-570

Asplund JM, Orskov ER, Hovell FD, Mac Leod NA (1985) The effect of intragastric infusion of glucose, lipids or acetate on fasting nitrogen excretion and blood metabolites in sheep. Br J Nutr 54, 189-195

Aurousseau B (1991) Élaboration des lipides corporels et valeur des carcasses des ruminants. Bull Tech CRVZ Theix INRA 45, 43-50

Aurousseau B, Thériez M, Daniel M (1973) Influence de la nature des matières grasses dans l'aliment d'allaitement sur le métabolisme lipidique de l'agneau de boucherie. Ann Biol Anim Biochim Biophys 13, 93-105

Aurousseau $B$, Vermorel $M$, Thériez $M$, Vezinhet A (1989a) Effects of substitution of tricaprilyn or coconut oil for tallow in milk replacers offered to preruminant lambs. Ann Zootech 38 , 49-59

Aurousseau B, Vermorel $M$, Thériez $M$ (1989b) Combined effects of tricaprilyn, coconut oil and amount of lipid, carbohydrate and protein on the digestibility of a milk replacer offered to the preruminant lamb on energy and nitrogen balance. Ann Zootech 38, 91-106

Beede DK, Collier RJ (1986) Potential nutritional strategies for intensively managed cattle during thermal stress. J Anim Sci 62, 543-554

Benevenga NJ, Steinman-Goldsworthy JK, Crenshaw TD, Odle $J$ (1989) Utilization of medium chain triglycerides by neonatal piglets: I. Effects on milk consumption and 
body fuel utilization. $J$ Anim Sci 67, 33313339

Bozzolo $G$, Bouillier-Oudot $M$, de Boisseson $E$, Ghassan M, Grasset D (1990) Influence des performances zootechniques sur les caractéristiques des tissus adipeux d'agneaux de bergerie, sevrés précocément et alimentés avec un régime à forte concentration énergétique. Ann Zootech 39, 77-94

Busboom JR, Miller GJ, Field RA, Crouse JD, Riley ML, Nelms GE, Ferell CL (1981) Characteristics of fat from heavy ram and wether lambs. J Anim Sci 52, 83-92

Cera KR, Mahan DC, Reinhart CA (1989) Appa* rent fat digestibilities and performance responses of post weaning swine fet diets supplemented with coconut oil, corn oil or tallow. $J$ Anim Sci 67, 2040-2047

Church DC (1979) Lipid utilization and requirement. In: Digestive Physiology and Nutrition of Ruminants : Nutrition, Oxford Press Publ, Portland, OR, vol 2, 167-186

Cottier $M$, Liquière $B$ (1986) La composition du lait des brebis Lacaune et ses variations. In: $11^{0}$ Journ Rech Ovine et Caprine, INRAITOVIC, SPEOC, Paris, 299-309

Dagnélie P (1977) Analyse statistique à plusieurs variables. Presses agronomiques de Gembloux, Gembloux

Dantzer R, Mormède $P$ (1983) Stress in farm animals: a need for reevaluation. $J$ Anim Sci $57,6-18$

Ferrell CL, Koong LJ, Nienaber JA (1986) Effect of previous nutrition on body composition and maintenance energy costs of growing lambs. Br J Nutr 56, 595-605

Frost SC, Wells MA (1981) A comparison of the utilization of medium and long chain fatty acids for oxidation and ketogenesis in the suckling rat: in vivo and in vitro studies. Arch Biochem Biophys 211, 357-366

Garton GA, Howell FD, Duncan WRH (1972) Influence of dietary volatile fatty acids on the fatty acid composition of lamb triglycerides with special reference to the effect of propionate on branched chain components. $\mathrm{Br} J$ Nutr 28, 409-416

Giger-Reverdin S, Aufrère J, Sauvant $D$, Demarquilly C, Vermorel M, Pochet S (1990) Prévision de la valeur énergétique des aliments composés pour les ruminants. INRA Prod Anim 3, 181-188

Leat WMF, Harrison PA (1975) Digestion, absorption and transport of lipids in the sheep. In: Digestion and Metabolism in the Ruminant (Mc Donald IW, Warner $\mathrm{ACl}$, eds) CSIRO, Sydney, Australia, 481-495

Marchello JA, Cramer DA, Miller LG (1967) Effect of ambiant temperature on certain ovine fat characteristics. J Anim Sci 26, 294-297

Odle J, Benevenga NJ, Crenshaw TD (1989) Utilization of medium chain triglycerides by neonatal piglets: II. Effects of even- and oddchain triglyceride consumption over the first 2 days of life on blood metabolites and urinary nitrogen excretion. J Anim Sci 67, 3340-3351

Sauvant D (1981) Prévision de la valeur énergétique des aliments concentrés et composés pour les ruminants. In: Prévision de la valeur nutritive des aliments des ruminants (Demarquilly $C$, ed) INRA Publ, Versailles, 237-258

Schanbacher BD, Hahn GL, Nienaber JA (1982) Effects of contrasting photoperiods and temperatures on performance traits of confinement-reared ewe lambs. J Anim Sci 55, 620626

Shelton M, Calhoun MC, Carpenter ZL (1972) The problem of soft oily lamb carcasses. Res Report PR 3025, Texas A \& M Univ, College Stn

Shetty PS (1990) Physiological mechanisms in the adaptative response of metabolic rates to energy restriction. Nutr Res Rev 3, 49-74

Sutton JD, Knight $R$, Mc Allan $A B$, Smith $R H$ (1983) Digestion and synthesis in the rumen of sheep given diets supplemented with free and protected oils. Br J Nutr 49, 419-432

Taylor Sr CS (1980) Genetic size-scaling rules in animal growth. Anim Prod 30, 161-165

Thompson JM, Parks JR (1985) Food intake, growth and body composition in Australian merino sheep selected for height and low weaning weight. 3. Energy balance. Anim Prod 40, 85-91

Turgeon OA, Brink DR, Bartle SJ, Klopfenstein TJ, Ferrell CL (1986) Effects of growth rate and compensatory growth on body composition in lambs. J Anim Sci 63, 770-780

Van Quackebeke E, Montagnon F, Cazes JP, Delmas G (1981) Alimentation des brebis laitières : influence de quelques caractéristi- 
ques des régimes alimentaires sur la quantité de lait produite et la composition du lait. In: La production laitière dans les espèces ovine et caprine. $6^{\theta}$ Journ Rech Ovine et Caprine INRA-ITOVIC, SPEOC, Paris, 271-310
Waghorn GC, Flux DS, Ulyatt MJ (1987) Effect of dietary protein and energy intakes on growth hormone, insulin, glucose tolerance and fatty acid synthesis in young wether sheep. Anim Prod 44, 143-152 